\title{
Combining Ability and Heterosis for Agronomic and Yield Traits in Indica and Japonica Rice Crosses
}

\author{
M. E. Yuga ${ }^{1}$, J. M. Kimani ${ }^{2}$, P. M. Kimani ${ }^{1}$, M. F. Olubayo ${ }^{1}$, J. W. Muthomi ${ }^{1}$ \& F. M. Nzuve ${ }^{1}$ \\ ${ }^{1}$ Department of Plant Science and Crop Protection, University of Nairobi, Nairobi, Kenya \\ ${ }^{2}$ Industrial Crops Research Centre, Kenya Agricultural and Livestock Research Organization (KALRO), \\ Kerugoya, Kenya \\ Correspondence: M. E. Yuga, Department of Plant Science and Crop Protection, University of Nairobi, P.O. Box \\ 29053-0625, Nairobi, Kenya. E-mail: myenoc@yahoo.com
}

Received: July 19, 2018 Accepted: August 30, $2018 \quad$ Online Published: November 15, 2018

doi:10.5539/jas.v10n12p92 URL: https://doi.org/10.5539/jas.v10n12p92

\begin{abstract}
Understanding genetic variability and mode of gene action for agronomic and yield related traits is important in formulation of effective rice breeding program for genetic enhancement of grain yield. Combining ability analysis and heterosis was conducted to identify yield associated traits from nine male indicas and three female japonicas, together with their $27 \mathrm{~F}_{1}$ hybrids. Four parental lines, including Basmati 370, Basmati 217, $\mathrm{K}_{2}-54$ and Komboka showed good general combining ability in days to $50 \%$ flowering, days to maturity, number of tillers plant $^{-1}$, number of spikelet's panicle ${ }^{-1}$, number of panicles plant ${ }^{-1}$, number of filled grains panicle ${ }^{-1}$, and grain yield. While the combine $\mathrm{K}_{2}-9 \times$ Komboka, $\mathrm{K}_{2}-9 \times$ Basmati 370, $\mathrm{K}_{2}-54 \times$ Dourado Precoce and $\mathrm{K}_{2}-54 \times$ Basmati 217 showed specific good for grain yield. The hybrids $\mathrm{K}_{2}-9 \times$ Basmati $370, \mathrm{~K}_{2}-8 \times$ Basmati $217, \mathrm{~K}_{2}-54 \times$ Basmati 217 and $\mathrm{K}_{2}-9 \times$ Komboka showed $20 \%$ excess in standard check variety, suggesting that they could be good breeding donors.
\end{abstract}

Keywords: Combining ability, earliness, gene action, heterosis, yield components, Oryza sativa

\section{Introduction}

Rice is the second most valued crop after maize in the world today (Syed \& Khaliq, 2008). The world rice requirement by 2050 will be 943.6 million tonnes which requires an annual increase of about 5.8 million tonnes from the present level of production (FAO, 2017). To achieve this target, it is necessary not only to utilize the existing upland and lowland land resources, but also develop upland rice varieties with high yield potential and durable resistance to both biotic and abiotic stresses (Dogara \& Jumare, 2014).

Rice forms a larger part of the diet for both urban and rural populations in Kenya. Kenya produces about 140,000 metric tonnes against a demand of over 540,000 metric tonnes per year (MoA, 2014). Unfortunately, rice yield per hectare is low $\left(<3.6 \mathrm{t} \mathrm{ha}^{-1}\right)$ because smallholder farmers rely on local cultivars with low yield potential, highly susceptible to bacterial leaf blight and rice blast (Kimani, 2010). Small holder farmers at Mwea irrigation scheme mainly grow Basmati rice under irrigated ecosystem. Basmati rice is mainly preferred by most consumers due to its aroma and good cooking qualities (Njiruh et al., 2013). However, yield of Basmati 370 is low $\left(3.0 \mathrm{t} \mathrm{ha}^{-1}\right)$. Upland rice varieties are not widely grown in Kenya despite their enormous potential in increasing national production (Kimani, 2010). This stagnation in productivity and associated high prices has led to decline in consumption of locally produced rice and increased imports from Pakistan, China, India and Vietnam (MoA, 2014).

Understanding the gene action for yield and yield related traits is a prerequisite for breeding high yielding locally adapted upland rice varieties (Dar et al., 2014). Various biometrical techniques designed to analyze genetic variability for yield related traits have been developed (Comstock \& Robinson, 1948; Griffing, 1956; Kempthrone, 1957; Kempthrone \& Curnow, 1961). These mating designs provide reliable information about general combining ability (GCA) and specific combining ability (SCA) of the parents and crosses. The differences in GCA are mainly due to additive gene action while the differences in SCA are attributed to non-additive gene effects (Fasahat et al., 2016). Estimation of GCA helps the breeder to identify parents with superior combining ability which may be hybridized to exploit heterosis, and also for development of breeding 
populations from which agronomically superior lines can be selected (Fasahat et al., 2016). Therefore, the knowledge of combining ability provides information on the nature and magnitude of gene effects that regulate grain yield and yield characters hence enabling the breeder to design an effective breeding method for genetic enhancement of grain yield and yield components (Dar et al., 2014).

Previous studies reported an increase in grain yield due to favourable heterosis for characters such as flag leaf area, number of spikelets per plant and number of filled grains per panicle (Vanaja \& Babu, 2004).

Positive heterosis is desirable for grain yield and negative heterosis is preferred for earliness. Heterosis is divided into heterobeltiosis, mid-parent heterosis, and standard heterosis (Alam et al., 2004). Standard heterosis is more important of the two levels of heterosis because it is aimed at developing desirable hybrids superior to the existing high yielding commercial varieties (Chaudhary, 1984). The objective of this study was to determine the combining ability and heterosis for agronomic traits and grain yield in indica and japonica rice crosses as a criterion for developing superior rice varieties.

\section{Materials and Methods}

\subsection{Study Location}

The study was conducted at Industrial Crops Research Centre, Mwea. The research station is located in Mwea Division, Kirinyaga County, Kenya. The area receives an average rainfall of about $850 \mathrm{~mm}$ over two seasons, with an average temperature ranging from $15.6{ }^{\circ} \mathrm{C}$ to $28.6^{\circ} \mathrm{C}$. The research station lies on a latitude $0^{\circ} 37^{\prime} \mathrm{S}$ and Longitude $37^{\circ} 20^{\prime} \mathrm{E}$ on an elevation of $1159 \mathrm{~m}$ above sea level. The soil type is nitosol with a PH of about 5.65. Monocropping is mainly practiced at the location. Water for irrigation is conveyed using partially lined canal and distributed through earthen canals (KARI, 2000).

\subsection{Rice Germplasm Used in the Study}

The pedigree and origin of the rice germplasm used in the study are presented in Table 1 . The male parents used in this study were indica type while the female parents were japonica type of rice. Four NERICAs, namely NERICA 1, 3, 10 and 11 were released in Kenya in 2006. NERICA 11 was the highest yielder $\left(5,451 \mathrm{~kg} \mathrm{ha}^{-1}\right)$ followed by NERICA $1\left(5,194 \mathrm{~kg} \mathrm{ha}^{-1}\right)$, NERICA $10\left(4,663 \mathrm{~kg} \mathrm{ha}^{-1}\right)$ and NERICA $3\left(4,270 \mathrm{~kg} \mathrm{ha}^{-1}\right)$ (WARDA, 2008). In addition, NERICA 1 is early maturing and well adapted to local conditions.

Table 1. Characteristics of parental lines used for hybridization

\begin{tabular}{|c|c|c|}
\hline Pedigree & ${ }^{8}$ Source & Primary selection criteria \\
\hline NERICA 1 & ARC & Slight aroma, high yielding, good grain quality, earliness, drought and blast resistant \\
\hline SARO 5 & ARI-KATRIN & Semi aromatic, high yielding (8-10 $\left.\mathrm{tha}^{-1}\right)$, earliness, high tillering (30-50 tillers) \\
\hline 08 FAN 10 & $\mathrm{ARC}$ & Semi aromatic, earliness, resistant to blast and high grain quality \\
\hline Basmati 370 & IRRI & $\begin{array}{l}\text { Aroma, high grain quality, high yield }\left(5.3 \mathrm{t} \mathrm{ha}^{-1}\right) \text {, resistant to rice yellow mottle virus and well } \\
\text { adapted to lowlands }\end{array}$ \\
\hline Basmati 217 & IRRI & $\begin{array}{l}\text { Aroma, high grain quality high yield }\left(4.6 \mathrm{t} \mathrm{ha}^{-1}\right) \text {, resistant to rice yellow mottle virus and } \\
\text { adapted to low nitrogen }\end{array}$ \\
\hline Dourado precoce & Kenya cultivar from Brazil & Moderate yielding $\left(3.5 \mathrm{tha}^{-1}\right)$, good cooking, high grain quality and adapted to highlands \\
\hline PAN 84 & KALRO-Mwea & $\begin{array}{l}\text { High yielding }\left(5.5 \mathrm{t} \mathrm{ha}^{-1}\right) \text {, good grain quality, tolerant to blast and well adapted to upland and } \\
\text { lowland areas }\end{array}$ \\
\hline IR 27-93-80-1-4 & IRRI & High yielding $\left(6 \mathrm{t} \mathrm{ha}^{-1}\right)$, earliness and good grain quality \\
\hline Komboka & IRRI & $\begin{array}{l}\text { High yielding }\left(6-7 \mathrm{tha}^{-1}\right) \text {, good grain quality, tolerant to diseases and well adapted to upland } \\
\text { and lowland areas }\end{array}$ \\
\hline $\mathrm{K}_{2}-9$ & RDA-KAFACI Korea & High yielding $\left(4-5 \mathrm{tha}^{-1}\right)$, earliness and well adapted to irrigated lowlands upland areas \\
\hline $\mathrm{K}_{2}-8$ & RDA-KAFACI Korea & High yielding $\left(6-7 \mathrm{tha}^{-1}\right)$, earliness and well adapted to irrigated lowlands upland areas \\
\hline $\mathrm{K}_{2}-54$ & RDA-KAFACI Korea & High yielding $\left(7 \mathrm{tha}^{-1}\right)$, earliness and well adapted to irrigated lowlands upland areas \\
\hline $\begin{array}{l}\text { Note. }{ }^{\S} \mathrm{ARC} \\
\text { Agricultural } \\
\text { Agricultural }\end{array}$ & frica Rice Centr & $\begin{array}{l}\text { ARI-KATRIN: Tanzania Agricultural Research Institute-Kilombero } \\
\text { istitute; IRRI: International Rice Research Institute; KALRO: Kenya } \\
\text { Organization; WARDA: West African Rice Development Association; }\end{array}$ \\
\hline
\end{tabular}




\subsection{Development of $F_{1}$ Hybrids}

The nine male indica and three female japonica parents were grown in a $5 \times 5 \mathrm{~m}^{2}$ hybridization nursery at the Industrial Crops Research Centre, Mwea during the short rain season between March and June 2016. The seeds were sown by direct seeding with two seeds per hill and the cultivars were replicated three times at an interval of 1,14 and 21 days to synchronize flowering and facilitate generation of enough $F_{1}$ seeds. Di-ammonium phosphate fertilizer was applied at a rate of $50 \mathrm{~kg} \mathrm{~N} \mathrm{ha}^{-1}$ and $40 \mathrm{~kg} \mathrm{Pha}^{-1}$ during planting to enhance plant growth and root vigour. The parents were crossed following North Carolina II mating design according to Comstock and Robinson (1952) to generate $27 \mathrm{~F}_{1}$ hybrids. Emasculation of the female was done using a vacuum emasculator (CAT No. 2027, Prasanthic Scientific, India) set at pressure of $15 \mathrm{kpa}$. The panicles of the male parents were cut using scissors before anther dehiscence and taken to the location of the female. The glassine bag was removed from the emasculated female and the pollinator panicles were gently shaken over the female panicle. The glassine bag was replaced over the female parent and the details of the male and female, emasculator and date of pollination were recorded on the tag, and attached to the female panicle (Coffman \& Herrera, 1980).

\subsection{Evaluation of $F_{1}$ Hybrids}

The $27 \mathrm{~F}_{1}$ hybrids and their parents were evaluated at Mwea Research Station during the cropping season of 2017. The trial was laid out in a randomized complete block design with three replications. The seeds were sown by direct seeding with an inter-row spacing of $20 \mathrm{~cm}$ and intra-row spacing of $20 \mathrm{~cm}$ with two seeds per hill. When the seedlings were 2-3 weeks old, gapping and thinning was carried out leaving one seedling per hill to ensure uniform plant density. Hand weeding was carried out at an interval of 20, 40 and 60 days after planting and DAP fertilizer (18:46:0) was applied at a rate of $50 \mathrm{~kg} \mathrm{~N}^{-1}$. The crop was top dressed twice at tillering with NPK (17:17:17) fertilizer at a rate of $47 \mathrm{~kg} \mathrm{~N} \mathrm{ha}^{-1}$ and at panicle initiation stage with calcium ammonium nitrates (CAN) fertilizer at a rate of $47 \mathrm{~kg} \mathrm{~N} \mathrm{ha}^{-1}$. The trial was irrigated twice every week while pesticide (Duduthrin $1.75 \mathrm{EC}$, Lambda-cyhalothrin $17.5 \mathrm{~g} / \mathrm{L}$ ) was applied every two weeks at a rate of $50 \mathrm{ml}$ per $20 \mathrm{~L}$ of water to control stem borer, fall armyworm and leaf hoppers.

\subsection{Determination of Agronomic and Yield Traits}

Agronomic and yield data was collected according to the procedure outlined in Standard Evaluation System (SES) for rice (IRRI, 2013). The quantitative and qualitative data was collected at vegetative, flowering, maturity and harvesting stages as follows:

(1) Days to $50 \%$ flowering: the number of days from sowing to when half of the plants in a plot and half of the tillers in these plants had flowered.

(2) Days to maturity: number of days from seedling emergence to when $85 \%$ of the spikelets were mature.

(3) Plant height: the height $(\mathrm{cm})$ of the plant from the plant base to the tip of the highest panicle excluding the awn was measured using a meter ruler.

(4) Number of tillers plant ${ }^{-1}$ : the numbers of tillers hill ${ }^{-1}$ were determined by counting all emerging shoots.

(5) Flag leaf length: the length $(\mathrm{cm})$ of the leaf immediately beneath the spike was measured from the collar to the nodal end of the leaf using a meter ruler.

(6) Panicle length: the length $(\mathrm{cm})$ of the panicle from the neck node base to the tip of the top most grain in the panicle was measured using a meter ruler.

(7) Number of fertile tillers plant ${ }^{-1}$ : the number of tillers bearing panicles at physiological maturity.

(8) Number of spikelet's panicle ${ }^{-1}$ : the number of spikelets on each panicle.

(9) Number of filled grains panicle ${ }^{-1}$ : two plants were sampled per plot and the panicles harvested, threshed and dried to $14 \%$ moisture content. The filled and the unfilled grains were separated using the floatation method (IRRI, 2013). The grains the sunk to the bottom of the beaker and those that floated were dried separately and the total number of the filled and unfilled grains were counted.

(10) Grain yield: the mature panicles were harvested, threshed, cleaned and dried to $14 \%$ moisture content. The threshed grains from each plant were weighed separately using electronic weigh balance (Model SP 401, OHAUS Lab balances, Amazon UK), recorded in grams and converted to $\mathrm{kg} \mathrm{ha}^{-1}$. The grain yield $\mathrm{plot}^{-1}$ was calculated using the formula described by Gomez (1972): 


$$
\text { Grain yield }=\frac{W}{n} \times N
$$

Where, $\mathrm{w}=$ weight of the grain from the harvested hills; $\mathrm{n}=$ number of harvested hills; $\mathrm{N}=$ total number of hills in a plot.

(11) Thousand grain weight: 1000 grains from each harvested plants were counted using electronic grain counter (Model AG64-100, Wagtech International, New York) and weighed after drying the grains to 14\% moisture content.

\subsection{Estimation of Combining Ability and Heterosis}

The agronomic and yield data was subjected to analysis of variance using analysis of genetic designs in $\mathrm{R}$ (AGD-R) version 3.0 (Gregorio et al., 2015). Their means were separated using the least significant differences (LSD) at $\mathrm{P} \leq 0.05$.

The variation between families was divided into component differences among males, females and that due to male $\times$ female interaction. The expectations of males and females in this design were equivalent to the general combining ability (GCA) and the interaction between males and females were equivalent to the specific combining ability (SCA). F-tests were done to examine the differences between males and females. The GCA and the SCA effects for the parents and their hybrids were calculated based on the statistical model by Comstock and Robinson (1952):

$$
\begin{gathered}
G C A \text { effect }\left(g_{1}\right)=\left(M s_{m}-M s_{m x f}\right) /[F r-\operatorname{Cov}(H s)]=1 / 4 \sigma^{2} A \\
G C A \text { effect }\left(g_{2}\right)=\left(M s_{f}-M s_{m x f}\right) /[M r-\operatorname{Cov}(H s)]=1 / 4 \sigma^{2} A \\
S C A \text { effect }\left(s_{1}\right)=\left(M s_{m x f}-M s_{e}\right) /[r-\operatorname{Cov}(F s)-2 \operatorname{Cov}(H s)]=1 / 4 \sigma^{2} D
\end{gathered}
$$

The ratio of GCA and SCA was computed according to Baker (1978):

$$
\frac{2 \sigma^{2} S C A}{2 \sigma^{2} G C A-\sigma^{2} S C A}
$$

Where, $\sigma^{2}$ GCA denotes general combining ability variance and $\sigma^{2}$ SCA for specific combining ability variance. A ratio closer to one reveals GCA action while a ratio of less than one predicts SCA action.

The magnitude of heterosis in the $\mathrm{F}_{1}$ hybrids was calculated in relation to the better parent rice variety as percent increase or decrease of the $F_{1}$ hybrids over the better parent. Better parent heterosis was computed according to Virmani et al. (1997):

$$
\frac{F_{1}-B P}{B P} \times 100 \%
$$

Where, $\mathrm{F}_{1}=$ mean performance of a single cross and $\mathrm{BP}=$ mean performance of the better parent.

\section{Results}

\subsection{Analysis of Variance}

General combining ability (GCA) was significant $(\mathrm{P} \leq 0.05)$ for days to $50 \%$ flowering, days to maturity, number of tillers, number of spikelet's panicle ${ }^{-1}$, number of productive tillers, number of filled grains panicle ${ }^{-1}$ and grain yield (Table 2). Specific combining ability (SCA) was significant $(\mathrm{P} \leq 0.05)$ for plant height, panicle length, flag leaf length and 1000 grain weight. The significant variation in the GCA and SCA variances suggested the importance of both additive and non-additive gene action in the expression of the yield traits.

GCA/SCA predictability ratio for duration to $50 \%$ flowering, days to maturity, number of tillers, number of spikelet's panicle $^{-1}$, number of productive tillers, number of filled grains panicle ${ }^{-1}$ and grain yield ratio was close to one suggesting the influence of additive gene action in the expression of these yield characters. However, the ratio for plant height, panicle length, flag leaf length and 1000 grain weight had a predictability ratio of less than one indicating the influence of non-additive action in the control of these traits. 
Table 2. Analysis of variance for combining ability of 12 parents and their $27 \mathrm{~F}_{1}$ hybrids for different yield traits

\begin{tabular}{|c|c|c|c|c|c|c|c|c|c|c|c|c|}
\hline $\begin{array}{l}\text { Source of } \\
\text { variance }\end{array}$ & df & $\begin{array}{l}50 \% \\
\text { Days to } \\
\text { flowering }\end{array}$ & $\begin{array}{l}\text { Days to } \\
\text { maturity }\end{array}$ & $\begin{array}{l}\text { Plant } \\
\text { height } \\
(\mathrm{cm})\end{array}$ & $\begin{array}{l}\text { Panicle } \\
\text { length } \\
(\mathrm{cm})\end{array}$ & $\begin{array}{l}\text { Flag leaf } \\
\text { length } \\
(\mathrm{cm})\end{array}$ & $\begin{array}{l}\text { Number } \\
\text { of tillers } \\
\text { plant }^{-1}\end{array}$ & $\begin{array}{l}\text { Number of } \\
\text { spikelets } \\
\text { panicle }^{-1}\end{array}$ & $\begin{array}{l}\text { Number } \\
\text { of fertile } \\
\text { tillers } \\
\text { plant }^{-1}\end{array}$ & $\begin{array}{l}\text { Number } \\
\text { of filled } \\
\text { grains } \\
\text { panicle }^{-1}\end{array}$ & $\begin{array}{l}\text { Grain } \\
\text { yield } \\
\left(\mathrm{kg} \mathrm{ha}^{-1}\right)\end{array}$ & $\begin{array}{l}1000 \\
\text { grain } \\
\text { weight }(\mathrm{g})\end{array}$ \\
\hline Replication & 2 & 15.27 & 8.78 & 0.70 & 0.31 & 0.64 & 3.49 & 0.60 & 4.93 & 152.94 & 0.44 & 0.05 \\
\hline Male & 8 & $1127.03 * *$ & $668.72 * *$ & $2805.78 * *$ & $51.52 * *$ & $183.20 * *$ & $1000.37 * *$ & $19.99 * *$ & $520.78^{* *}$ & $1379.81 * *$ & $6.86 * *$ & $5.41 * *$ \\
\hline Female & 2 & $90.83 * *$ & $194.78 * *$ & $98.26 * *$ & 0.75 & $82.23 * *$ & $149.86^{* *}$ & $2.68 * *$ & $78.37 * *$ & $1068.38^{* *}$ & $0.63 * *$ & $0.75^{*}$ \\
\hline Male $\times$ Female & 16 & $574.98 * *$ & $275.14^{* *}$ & $612.63^{* *}$ & $13.45 * *$ & $103.30^{* *}$ & $433.03^{* *}$ & $4.12 * *$ & $302.70^{* *}$ & $857.38^{* *}$ & $2.42 * *$ & $4.44 * *$ \\
\hline Error & 52 & 12.77 & 7.80 & 1.54 & 0.42 & 1.10 & 8.93 & 0.32 & 9.48 & 18.36 & 0.10 & 0.18 \\
\hline$\sigma^{2} \mathrm{~m}$ & - & 67.32 & 44.72 & 243.68 & 4.23 & 8.88 & 66.53 & 1.78 & 27 & 58.05 & 0.52 & 0.15 \\
\hline$\sigma^{2} f$ & - & $7.72 \mathrm{E}-13$ & -0.16 & -0.31 & -0.01 & -0.13 & $1.28 \mathrm{E}-14$ & $1.99 \mathrm{E}-15$ & -0.23 & 7.81 & -0.01 & -0.22 \\
\hline$\sigma^{2} \mathrm{~m} \times \mathrm{f}$ & - & 169.47 & 86.14 & 203.70 & 4.34 & 34.07 & 130.88 & 1.21 & 89.43 & 279.67 & 0.71 & 1.28 \\
\hline$\sigma^{2} \mathrm{~A}$ & - & 926.44 & 509.68 & 524.33 & 8.86 & 18.95 & 769.18 & 11.43 & 457.43 & 1354.66 & 4.73 & 5.14 \\
\hline$\Sigma^{2} \mathrm{D}$ & - & 677.88 & 344.54 & 814.80 & 17.36 & 136.27 & 523.52 & 4.85 & 357.73 & 1118.70 & 2.83 & 5.74 \\
\hline$\Sigma^{2} \mathrm{~A} / \Sigma^{2} \mathrm{D}$ & - & 1.4 & 1.5 & 0.6 & 0.5 & 0.1 & 1.5 & 1.35 & 1.27 & 1.21 & 1.67 & 0.89 \\
\hline
\end{tabular}

Note. $\mathrm{df}=$ degrees of freedom, ${ }^{*}$ Significance at $5 \%$ level of probability, ${ }^{* *}$ Significance at $1 \%$ level of probability, $\sigma^{2} \mathrm{~m}=$ Male variance, $\sigma^{2} \mathrm{f}=$ Female variance, $\sigma^{2} \mathrm{~m} \times \mathrm{f}=$ Interaction effect of male and female, $\sigma^{2} \mathrm{~A}=$ Additive variance, $\sigma^{2} \mathrm{D}=$ Dominance variance.

\subsection{General Combining Ability (GCA) Effects of the Parents}

The general combining ability effects for agronomic and yield traits are presented in Table 3. Komboka, SARO 5, Basmati 217 and $\mathrm{K}_{2}-54$ took longer to reach $50 \%$ flowering while NERICA 1, PAN 84, Basmati 370, IR-27-93-80-1, Dourado precoce and $\mathrm{K}_{2}-9$ took shorter days to 50\% flowering due to their negative GCA effects. Komboka, Basmati 217 and SARO 5 were late maturing while NERICA 1, PAN 84, Dourado precoce and $\mathrm{K}_{2}-54$ displayed negative GCA effects and were early maturing. Basmati 370, Basmati 217 and PAN 84 were tall plants because they exhibited positive and significant GCA effects for plant height while IR-27-93-80-1, SARO 5, 08FAN 10, NERICA 1, Komboka and $\mathrm{K}_{2}-8$ with negative GCA effects were short statured plants. Basmati 370 and Basmati 217 had long panicles while $\mathrm{K}_{2}-8$, Komboka, Dourado precoce, PAN 84 and NERICA 1 exhibited short panicles. Basmati 217, PAN 84, Basmati 370 and $\mathrm{K}_{2}-54$ had long flag leaf while Dourado precoce, Komboka, $\mathrm{K}_{2}-8, \mathrm{~K}_{2}-9$, SARO 5 and NERICA 1 exhibited short flag leaf. Komboka, Basmati 217, Basmati 370 and $\mathrm{K}_{2}-54$ had more number of tillers plant ${ }^{-1}$ while Dourado precoce, PAN 84, SARO 5, NERICA 1 and $\mathrm{K}_{2}-9$ had fewer tillers. SARO 5 and Komboka had more spikelets per panicle while Basmati 370 had the least spikelets per panicle. Komboka, Basmati 217, Basmati 370 and $\mathrm{K}_{2}-9$ had more panicles per plant while Dourado precoce, PAN 84, SARO 5 and NERICA 1 had fewer panicles per plant. Komboka, Basmati 217, IR-27-93-80-1, PAN 84 and $\mathrm{K}_{2}-9$ had more filled grains per panicle while 08FAN 10, NERICA 1, Basmati 370, Dourado precoce and $\mathrm{K}_{2}-54$ displayed fewer filled grains per panicle. Basmati 370, Komboka, Basmati 217 and $\mathrm{K}_{2}-54$ were high grain yielders while SARO 5, $\mathrm{K}_{2}-8$, 08FAN 10, NERICA 1 and IR-27-93-80-1 had low grain yield. Basmati 217, PAN 84, Dourado precoce and $\mathrm{K}_{2}-8$ had heavy 1000 grain weight while IR-27-93-80-1, 08FAN 10, Basmati 370 and $\mathrm{K}_{2}-54$ had lighter 1000 grain weight.

\subsection{Specific Combining Ability Effects of $27 F_{1}$ Hybrids for Agronomic and Yield Traits}

The specific combining ability effects for agronomic and yield related traits are presented in Table 4 . The $\mathrm{F}_{1}$ hybrids $\mathrm{K}_{2}-9 \times$ Komboka, $\mathrm{K}_{2}-9 \times$ Basmati $370, \mathrm{~K}_{2}-54 \times$ Dourado precoce, and $\mathrm{K}_{2}-54 \times$ Basmati 217 had high

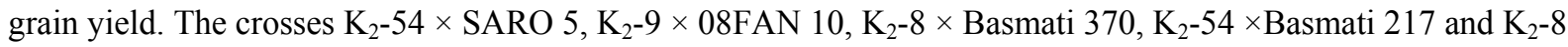
$\times$ Komboka were late maturing while $\mathrm{K}_{2}-54 \times \mathrm{IR}-27-93-80-1, \mathrm{~K}_{2}-8 \times 08 \mathrm{FAN} 10, \mathrm{~K}_{2}-54 \times$ Basmati 370 and $\mathrm{K}_{2}-54 \times$ Dourado precoce were early maturing. $\mathrm{K}_{2}-54 \times$ Basmati $370, \mathrm{~K}_{2}-54 \times$ Basmati $217, \mathrm{~K}_{2}-9 \times$ Dourado precoce and $\mathrm{K}_{2}-9 \times$ Komboka had tall plants while $\mathrm{K}_{2}-54 \times$ Dourado precoce and $\mathrm{K}_{2}-8 \times$ Komboka were short statured plants. $\mathrm{K}_{2}-8 \times$ Basmati $370, \mathrm{~K}_{2}-54 \times$ Basmati 217 and $\mathrm{K}_{2}-9 \times$ Dourado precoce had long panicles while $\mathrm{K}_{2}-9 \times 08$ FAN 10, $\mathrm{K}_{2}-54 \times$ Dourado precoce and $\mathrm{K}_{2}-54 \times$ Komboka displayed short panicles. The hybrids $\mathrm{K}_{2}-54$ $\times$ Basmati 217, $\mathrm{K}_{2}-54 \times$ Basmati $370, \mathrm{~K}_{2}-8 \times 08$ FAN 10 and $\mathrm{K}_{2}-9 \times$ Komboka exhibited more tillers plant $^{-1}$ while $\mathrm{K}_{2}-54 \times$ Dourado precoce, $\mathrm{K}_{2}-9 \times$ PAN 84, $\mathrm{K}_{2}-8 \times$ Basmati 217 and $\mathrm{K}_{2}-8 \times$ SARO 5 had fewer tillers plant $^{-1} . \mathrm{K}_{2}-54 \times$ Komboka, $\mathrm{K}_{2}-54 \times$ PAN 84 and $\mathrm{K}_{2}-9 \times$ Basmati $_{370}$ had more spikelets panicle ${ }^{-1}$ while $\mathrm{K}_{2}-54 \times$ Basmati 370 had the least spikelets panicle ${ }^{-1}$. The hybrids $\mathrm{K}_{2}-9 \times$ Komboka, $\mathrm{K}_{2}-54 \times$ Basmati 217 and $\mathrm{K}_{2}-54 \times$ Basmati 370 had more panicles plant ${ }^{-1}$ while $\mathrm{K}_{2}-8 \times$ SARO 5, $\mathrm{K}_{2}-54 \times$ Dourado precoce and $\mathrm{K}_{2}-54 \times$ Komboka had fewer panicles plant ${ }^{-1}$. The combine $\mathrm{K}_{2}-54 \times$ SARO 5, $\mathrm{K}_{2}-8 \times$ Basmati 370, $\mathrm{K}_{2}-9 \times$ Basmati 370 and $\mathrm{K}_{2}-9 \times$ Komboka had high number of filled grains per panicle while $\mathrm{K}_{2}-54 \times$ Dourado precoce, $\mathrm{K}_{2}-8 \times 08$ FAN 10 and 
$\mathrm{K}_{2}-8 \times$ NERICA 1 had the least filled grains per panicle. $\mathrm{K}_{2}-9 \times$ Dourado precoce, $\mathrm{K}_{2}-54 \times$ Dourado precoce, $\mathrm{K}_{2}-54 \times$ PAN 84, $\mathrm{K}_{2}-8 \times$ Basmati 217, $\mathrm{K}_{2}-8 \times$ Basmati 370 and $\mathrm{K}_{2}-8 \times$ NERICA 1 had high 1000 grain weight while $\mathrm{K}_{2}-9 \times$ Komboka, $\mathrm{K}_{2}-9 \times$ PAN 84, $\mathrm{K}_{2}-54 \times$ Basmati 370 and $\mathrm{K}_{2}-54 \times$ SARO 5 had lighter 1000 grain weight.

Table 3. General combining ability (GCA) effects of 12 rice parents for agronomic and yield components

\begin{tabular}{|c|c|c|c|c|c|c|c|c|c|c|c|}
\hline Genotype & $\begin{array}{l}50 \% \\
\text { Days to } \\
\text { flowering }\end{array}$ & $\begin{array}{l}\text { Days to } \\
\text { maturity }\end{array}$ & $\begin{array}{l}\text { Plant } \\
\text { height } \\
(\mathrm{cm})\end{array}$ & $\begin{array}{l}\text { Panicle } \\
\text { length } \\
(\mathrm{cm})\end{array}$ & $\begin{array}{l}\text { Flag } \\
\text { leaf } \\
\text { length } \\
(\mathrm{cm}) \\
\end{array}$ & $\begin{array}{l}\text { Number } \\
\text { of tillers } \\
\text { plant }^{-1}\end{array}$ & $\begin{array}{l}\text { Number of } \\
\text { spikelet's } \\
\text { panicle }^{-1}\end{array}$ & $\begin{array}{l}\text { Number } \\
\text { of fertile } \\
\text { tillers } \\
\text { plant }^{-1}\end{array}$ & $\begin{array}{l}\text { Number } \\
\text { of filled } \\
\text { grains } \\
\text { panicle }^{-1}\end{array}$ & $\begin{array}{l}\text { Grain } \\
\text { yield } \\
\left(\mathrm{kg} \mathrm{ha}^{-1}\right)\end{array}$ & $\begin{array}{l}1000 \\
\text { grain } \\
\text { weight }(\mathrm{g})\end{array}$ \\
\hline \multicolumn{12}{|l|}{ Males } \\
\hline NERICA 1 & -12.49 & -6.59 & -9.15 & -1.13 & -1.84 & -4.46 & -0.73 & -4.37 & -13.16 & -457.2 & 0.23 \\
\hline IR-27-93-80-1 & -6.6 & -1.81 & -14.85 & -0.36 & -5.73 & 1.46 & 0.51 & 3.96 & $9.95 * *$ & -468.1 & -0.87 \\
\hline SARO 5 & $14.84 *$ & $7.85^{*}$ & -10.13 & -1.21 & -5.62 & -2.07 & $2.39^{* *}$ & -6.15 & 4.28 & -506.8 & -0.34 \\
\hline 08 FAN 10 & -3.6 & -3.59 & -9.42 & -1.55 & -0.51 & 1.99 & -0.82 & 0.96 & -15.49 & -368.3 & -0.81 \\
\hline Basmati 370 & -7.72 & 0.85 & $24.71 * *$ & $2.37 *$ & $3.27 *$ & $4.38 *$ & $-1.45^{* *}$ & $5.07 *$ & -4.83 & $1017.9^{* *}$ & -0.24 \\
\hline Basmati 217 & $11.51^{*}$ & $11.19^{*}$ & $16.69^{*}$ & $3.81 * *$ & $7.05^{*}$ & $5.45 *$ & -0.91 & $8.52 *$ & $10.17 * *$ & $902.5^{* *}$ & $1.08 * *$ \\
\hline PAN 84 & -8.27 & -11.04 & $8.23 *$ & -0.36 & $5.49 *$ & -8.85 & 0.07 & -9.59 & $7.73 *$ & -78.04 & $0.31^{*}$ \\
\hline Dourado precoce & -4.6 & -8.93 & 5.11 & -0.53 & -0.06 & -7.86 & -0.2 & -9.15 & -15.27 & 70.8 & $1.25^{* *}$ \\
\hline Komboka & $16.95^{*}$ & $12.07 *$ & -11.2 & -1.04 & -2.06 & $9.97 *$ & $1.14^{*}$ & $10.74 * *$ & $16.62 * *$ & $66.5^{*}$ & -0.61 \\
\hline \multicolumn{12}{|l|}{ Females } \\
\hline $\mathrm{K}_{2}-8$ & 0.73 & 0.37 & -2.19 & -0.17 & -1.1 & -2.27 & -0.36 & -1.7037 & -3.2 & -160 & $0.16^{* *}$ \\
\hline $\mathrm{K}_{2}-54$ & 1.36 & -2.85 & 1.33 & 0.01 & $2.01 * *$ & $2.43 * *$ & 0.2 & $-1.40 \mathrm{E}-14$ & -4.05 & $140 * *$ & -0.17 \\
\hline $\mathrm{K}_{2}-9$ & -2.09 & 2.48 & 0.85 & 0.16 & -0.91 & -0.16 & 0.12 & $1.703 * *$ & $7.25 * *$ & 200 & 0.01 \\
\hline
\end{tabular}

Note. ${ }^{*}$ Significance at $5 \%$ level of probability, ${ }^{* *}$ Significance at $1 \%$ level of probability.

Table 4. Specific combining ability (SCA) effects of $27 \mathrm{~F}_{1}$ hybrids for different agronomic and yield traits

\begin{tabular}{|c|c|c|c|c|c|c|c|c|c|c|c|}
\hline $\begin{array}{l}\text { Crosses } \\
\text { Combination }\end{array}$ & $\begin{array}{l}50 \% \\
\text { Days to } \\
\text { flowering }\end{array}$ & $\begin{array}{l}\text { Days to } \\
\text { maturity }\end{array}$ & $\begin{array}{l}\text { Plant } \\
\text { height } \\
(\mathrm{cm})\end{array}$ & $\begin{array}{l}\text { Panicle } \\
\text { length } \\
(\mathrm{cm})\end{array}$ & $\begin{array}{l}\text { Flag } \\
\text { leaf } \\
\text { length } \\
(\mathrm{cm})\end{array}$ & $\begin{array}{l}\text { Number } \\
\text { of tillers } \\
\text { plant }^{-1}\end{array}$ & $\begin{array}{l}\text { Number } \\
\text { of spikelet' } \\
\text { panicle }^{-1}\end{array}$ & $\begin{array}{l}\text { Number } \\
\text { sof panicles } \\
\text { plant }^{-1}\end{array}$ & $\begin{array}{l}\text { Number } \\
\text { of filled } \\
\text { grains } \\
\text { panicle }{ }^{-1}\end{array}$ & $\begin{array}{l}\text { Grain } \\
\text { yield } \\
\left(\mathrm{kg} \mathrm{ha}^{-1}\right)\end{array}$ & $\begin{array}{l}1000 \\
\text { grain } \\
\text { weight }(\mathrm{g})\end{array}$ \\
\hline $\mathrm{K}_{2}-8 \times$ NERICA1 & -9.54 & -3.95 & -1.03 & -0.33 & -2.65 & -3.04 & -1.19 & -4.83 & $-28.37 * * *$ & -208.2 & $0.98 * *$ \\
\hline $\mathrm{K}_{2}-54 \times$ NERICA 1 & -4.01 & -1.36 & -2.36 & -0.65 & 2.62 & -3.04 & 0.34 & -0.96 & 7.68 & 540 & -0.36 \\
\hline $\mathrm{K}_{2}-9 \times$ NERICA 1 & -3.36 & -2.33 & -3.36 & -0.01 & -2.98 & -2.71 & 0.34 & -0.96 & -3.31 & $-1038.6^{*}$ & -0.14 \\
\hline $\mathrm{K}_{2}-8 \times$ IR-27-93-80-1 & -4.5 & 1.67 & 0.67 & -0.11 & 1.38 & 5.52 & -0.5 & 5.58 & 3.84 & -6.66 & $-0.84 *$ \\
\hline $\mathrm{K}_{2-} 54 \times \mathrm{IR}-27-93-80-1$ & -9.7 & $-11.27 * * *$ & -2.99 & 0.22 & $-5.54 * * *$ & -0.68 & 0.73 & -0.86 & 4.66 & -568.2 & $-1.02 * * *$ \\
\hline $\mathrm{K}_{2}-9 \times$ IR-27-93-80-1 & 5.26 & 7.5 & -8.64 & -0.43 & $-5.21 *$ & -1.98 & 0.12 & 1.4 & 9.65 & -136 & 0.01 \\
\hline $\mathrm{K}_{2}-8 \times$ SARO5 & -1.43 & -0.74 & -9.7 & -1.54 & $-10.53 * * *$ & -8.64 & 0.85 & $-11.43 * * *$ & -7.76 & -740 & 0.14 \\
\hline $\mathrm{K}_{2}-54 \times$ SARO5 & $22.95 * * *$ & $11.55^{* * *}$ & 9.25 & 0.39 & $9.58 * * *$ & 9.62 & 0.54 & 3.06 & $19.15^{* * *}$ & 624.9 & $-1.07 * * *$ \\
\hline $\mathrm{K}_{2}-9 \times$ SARO5 & -1.43 & -1.71 & -7.04 & 0.07 & $-8.23 * * *$ & -5.05 & 0.24 & -1.13 & -3.58 & -655.7 & 0.2 \\
\hline $\mathrm{K}_{2}-8 \times$ FAN10 & -5.42 & $-13.14 * * *$ & 4.55 & 1.04 & 1.37 & $10.86^{*}$ & -0.49 & 7.26 & $-17.39 * * *$ & -575.9 & -0.48 \\
\hline $\mathrm{K}_{2}-54 \times \mathrm{FAN} 10$ & -2.82 & -4.08 & -3.09 & 0.08 & $5.33^{*}$ & -5.76 & 0.12 & -1.11 & 2.34 & 266.6 & $-1.20 * * *$ \\
\hline $\mathrm{K}_{2}-9 \times$ FAN10 & 3.36 & $13.06^{* * *}$ & -8.41 & $-2.49 * * *$ & $-7.53 * * *$ & -1.2 & -0.19 & -4.66 & -13.21 & -552.1 & -0.05 \\
\hline $\mathrm{K}_{2}-8 \times$ Basmati 370 & $12.02 *$ & $10.14 * * *$ & -7.88 & $2.09 *$ & 3.65 & -9.73 & 0.08 & -3.93 & $16.17 * * *$ & 875.9 & $1.82 * * *$ \\
\hline $\mathrm{K}_{2}-54 \times$ Basmati 370 & $-27.98 * * *$ & $-17.04 * * *$ & $30.35 * * *$ & 1.77 & 0.02 & $24.82 * * *$ & $-2.37 * * *$ & $12.49 * * *$ & $-39.45 * * *$ & -471.8 & $-2.24 * * *$ \\
\hline $\mathrm{K}_{2}-9 \times$ Basmati 370 & 5.52 & 7.88 & -4.22 & -1.77 & 1.67 & -6.47 & $1.30^{*}$ & -0.71 & $14.47^{*}$ & $1121.9^{*}$ & -0.09 \\
\hline $\mathrm{K}_{2}-8 \times$ Basmati 217 & -1.64 & 1.52 & -1.87 & -1.56 & -1.32 & -6.86 & -1.02 & -7.42 & 1.15 & 338.2 & $1.24 * * *$ \\
\hline $\mathrm{K}_{2}-54 \times$ Basmati 217 & $26.00 * * *$ & $14.14 * * *$ & $18.40 * * *$ & $3.91 * * *$ & $10.88 * * *$ & $17.59^{* * *}$ & 0.2 & $15.44 * * *$ & 11.1 & $986.5^{*}$ & 0.17 \\
\hline $\mathrm{K}_{2}-9 \times$ Basmati 217 & -8.79 & -2.69 & -4.2 & 1.01 & 1.97 & -0.01 & 0.2 & 5.14 & 6.31 & 28.4 & $0.90 * *$ \\
\hline $\mathrm{K}_{2}-8 \times \mathrm{PAN} 84$ & -2.65 & -2.97 & 8.56 & -0.75 & 2.66 & -2.98 & -0.7 & -0.22 & 8.91 & 702.83 & -0.13 \\
\hline $\mathrm{K}_{2}-54 \times \mathrm{PAN} 84$ & -8.18 & -8.15 & -5.07 & 0.54 & -0.3 & -7.87 & $1.44 * * *$ & -7.63 & -5.61 & -320.52 & $1.91 * * *$ \\
\hline $\mathrm{K}_{2}-9 \times \mathrm{PAN} 84$ & -0.37 & -1.68 & 2.58 & -0.11 & $6.62 * * *$ & -6.57 & -0.7 & -6.98 & 10.8 & 204.61 & $-1.13 * * *$ \\
\hline $\mathrm{K}_{2}-8 \times$ Dourado precoce & -5.22 & -1.29 & 4.36 & -0.58 & 1.83 & 0.28 & 0.77 & 2.16 & -4.43 & $-848.2 *$ & -0.52 \\
\hline $\mathrm{K}_{2}-54 \times$ Dourado precoce & 5.51 & $-12.29 * * *$ & $-18.91 * * *$ & $-3.16 * * *$ & $-6.41 * * *$ & -8.19 & -1.07 & $-10.72 * * *$ & $-35.58 * * *$ & $1090.4^{*}$ & $1.56^{* * *}$ \\
\hline $\mathrm{K}_{2}-9 \times$ Dourado precoce & -6.52 & 3.24 & $18.32 * * *$ & $3.27 * * *$ & 4.47 & -7.54 & 0.16 & -5.57 & 12.15 & -136 & $1.63 * * *$ \\
\hline $\mathrm{K}_{2}-8 \times$ Komboka & $24.77 * * *$ & $11.00 * * *$ & $-17.27 * * *$ & 0.23 & $-6.18 * * *$ & -5.41 & -0.76 & -1.98 & 5.29 & -778.7 & $-0.84 *$ \\
\hline $\mathrm{K}_{2}-54 \times$ Komboka & 10.14 & 3.59 & -13.62 & $-2.99 * * *$ & 1.73 & -5.09 & $1.99 * * *$ & $-9.71 * * *$ & 7.08 & -285.2 & 0.76 \\
\hline $\mathrm{K}_{2-}-9 \times$ Komboka & $-11.97 * * *$ & -1.59 & $22.62 * * *$ & 1.84 & 1.07 & $30.11^{* * *}$ & -0.46 & $28.29 * * *$ & $17.95 * * *$ & $1995.1^{* * *}$ & $-1.21 * * *$ \\
\hline
\end{tabular}

Note. ${ }^{*}$ Significance at $5 \%$ level of probability, ${ }^{* *}$ Significance at $1 \%$ level of probability, ${ }^{* * *}$ Significance at $\mathrm{P}$ $\leq 0.001$. 


\subsection{Heterosis for Agronomic and Yield Traits}

There was marked variation in heterosis for yield and yield related traits among the $\mathrm{F}_{1}$ progenies studied (Table 5). High parent heterosis for grain yield varied from $-1 \%\left(\mathrm{~K}_{2}-54 \times \mathrm{SARO} 5\right)$ to $31 \%\left(\mathrm{~K}_{2}-9 \times\right.$ Basmati 370$)$. The hybrids $\mathrm{K}_{2}-9 \times$ Basmati 370, $\mathrm{K}_{2}-9 \times$ Komboka, $\mathrm{K}_{2}-8 \times$ Basmati $217, \mathrm{~K}_{2}-54 \times$ Basmati 217 and K2-54 $\times$ Dourado precoce were high grain yielders while $\mathrm{K}_{2}-9 \times$ NERICA $1, \mathrm{~K}_{2}-9 \times$ SARO 5 and $\mathrm{K}_{2}-9 \times$ PAN 84 had negative heterosis for grain yield. High parent heterosis for number of tillers per plant varied from $-2 \%\left(\mathrm{~K}_{2}-8 \times\right.$ Dourado precoce) to $90 \%\left(\mathrm{~K}_{2}-9 \times\right.$ Komboka) with $\mathrm{K}_{2}-9 \times$ Komboka, $\mathrm{K}_{2}-54 \times$ Basmati 217 and $\mathrm{K}_{2}-54 \times$ Basmati 370 exhibiting the highest while $\mathrm{K}_{2}-54 \times$ Dourado precoce and K2-9 $\times$ PAN 84 had negative heterosis for number of tillers. The hybrids $\mathrm{K}_{2}-8 \times$ Basmati 370, $\mathrm{K}_{2}-54 \times$ Basmati 370, $\mathrm{K}_{2}-9 \times$ Basmati $217, \mathrm{~K}_{2}-9 \times$ Basmati 217 and $\mathrm{K}_{2}-8 \times$ Basmati 370 showed high parent heterosis for panicle length while $\mathrm{K}_{2}-54 \times$ Komboka and $\mathrm{K}_{2}-54 \times$ Dourado precoce exhibited negative heterosis for panicle length. The hybrids $\mathrm{K}_{2}-54 \times$ Basmati $370, \mathrm{~K}_{2}-9 \times$ Komboka and $\mathrm{K}_{2}-9 \times$ PAN 84 had high parent heterosis for filled grains per panicle while $\mathrm{K}_{2}-8 \times$ Basmati 370 and $\mathrm{K}_{2}-8 \times 08$ FAN 10 had negative heterosis. The crosses $\mathrm{K}_{2}-54 \times$ Basmati 217, $\mathrm{K}_{2}-8 \times$ Komboka and $\mathrm{K}_{2}-54 \times$ SARO 5 expressed high parent heterosis for days to maturity while $\mathrm{K}_{2}-54 \times$ Basmati $370, \mathrm{~K}_{2}-54 \times$ Dourado precoce were early maturing over the better parent (NERICA 1).

Table 5. Heterosis for agronomic and yield components in indica and japonica rice crosses

\begin{tabular}{|c|c|c|c|c|c|c|c|c|c|c|c|c|c|c|}
\hline \multirow[b]{2}{*}{$\begin{array}{l}\text { Cross } \\
\text { Combination }\end{array}$} & \multicolumn{4}{|c|}{ Earliness } & \multicolumn{7}{|c|}{ Yield components } & \multicolumn{3}{|c|}{ Grain yield } \\
\hline & $\begin{array}{l}50 \% \\
\text { Days to } \\
\text { flowering }\end{array}$ & $\begin{array}{l}\text { Better } \\
\text { parent }\end{array}$ & $\begin{array}{l}\text { Days to } \\
\text { maturity }\end{array}$ & $\begin{array}{l}\text { Better } \\
\text { parent }\end{array}$ & $\begin{array}{l}\text { Panicle } \\
\text { length }\end{array}$ & $\begin{array}{l}\text { Better } \\
\text { parent }\end{array}$ & $\begin{array}{l}\text { Number } \\
\text { of tillers } \\
\text { plant }^{-1}\end{array}$ & $\begin{array}{l}\text { Better } \\
\text { parent }\end{array}$ & $\begin{array}{l}\text { Number } \\
\text { of panicles } \\
\text { plant }^{-1}\end{array}$ & $\begin{array}{l}\text { Better } \\
\text { parent }\end{array}$ & $\begin{array}{l}\text { Filled } \\
\text { grains } \\
\text { panicle }^{-1}\end{array}$ & $\begin{array}{l}\text { Better } \\
\text { parent }\end{array}$ & $\begin{array}{l}\text { Grain } \\
\text { yield } \\
\text { plot }^{-1}\end{array}$ & $\begin{array}{l}\text { Better } \\
\text { parent } \\
\left(\mathrm{kg} \mathrm{ha}^{-1}\right)\end{array}$ \\
\hline K9 9 NERICA 1 & $-2 \%$ & 82 & $8 \%$ & 112 & $-13 \%$ & 23 & $-7 \%$ & 39 & $-19 \%$ & 43 & $-6 \%$ & 127 & $-28 \%$ & 4,360 \\
\hline K8 $\times$ NERICA 1 & $-24 \%$ & 84 & $1 \%$ & 124 & $-9 \%$ & 21 & $0 \%$ & 41 & $-14 \%$ & 28 & $-15 \%$ & 110 & $-14 \%$ & 4,280 \\
\hline K54 × NERICA 1 & $2 \%$ & 82 & $12 \%$ & 118 & $-9 \%$ & 20 & $-7 \%$ & 40 & $-16 \%$ & 30 & $-13 \%$ & 120 & $-10 \%$ & 4,620 \\
\hline K9 9 SARO 5 & $22 \%$ & 86 & $18 \%$ & 131 & $-13 \%$ & 19 & $0 \%$ & 36 & $-19 \%$ & 27 & $-8 \%$ & 117 & $-32 \%$ & 3,643 \\
\hline K8 $\times$ SARO 5 & $20 \%$ & 85 & $20 \%$ & 130 & $-13 \%$ & 20 & $-7 \%$ & 37 & $-37 \%$ & 26 & $-9 \%$ & 120 & $-32 \%$ & 3,639 \\
\hline K54 × SARO 5 & $51 \%$ & 99 & $28 \%$ & 129 & $-9 \%$ & 21 & $34 \%$ & 41 & $-7 \%$ & 25 & $16 \%$ & 121 & $-1 \%$ & 3,680 \\
\hline K9 908 FAN 10 & $15 \%$ & 94 & $24 \%$ & 139 & $-26 \%$ & 18 & $22 \%$ & 48 & $-26 \%$ & 36 & $-17 \%$ & 106 & $-29 \%$ & 3,967 \\
\hline $\mathrm{K} 8 \times 08 \mathrm{FAN} 10$ & $4 \%$ & 82 & $0 \%$ & 138 & $-4 \%$ & 21 & $44 \%$ & 47 & $12 \%$ & 38 & $-22 \%$ & 109 & $-14 \%$ & 3,887 \\
\hline K54 × 08 FAN 10 & $7 \%$ & 90 & $8 \%$ & 140 & $-9 \%$ & 19 & $7 \%$ & 49 & $-9 \%$ & 40 & $-8 \%$ & 110 & $-11 \%$ & 3,680 \\
\hline K9 $\times$ Basmati 370 & $15 \%$ & 82 & $21 \%$ & 142 & $0 \%$ & 29 & $17 \%$ & 41 & $7 \%$ & 37 & $8 \%$ & 148 & $31 \%$ & 5,470 \\
\hline $\mathrm{K} 8 \times$ Basmati 370 & $22 \%$ & 85 & $23 \%$ & 140 & $17 \%$ & 30 & $2 \%$ & 40 & $-12 \%$ & 40 & $-35 \%$ & 142 & $18 \%$ & 5,281 \\
\hline K54 $\times$ Basmati 370 & $-32 \%$ & 106 & $-8 \%$ & 141 & $13 \%$ & 28 & $54 \%$ & 42 & $2 \%$ & 38 & $35 \%$ & 150 & $-13 \%$ & 5,681 \\
\hline K9 $\times$ Basmati 217 & $10 \%$ & 82 & $18 \%$ & 136 & $18 \%$ & 29 & $29 \%$ & 62 & $14 \%$ & 53 & $6 \%$ & 127 & $0 \%$ & 4,877 \\
\hline K8 $\times$ Basmati 217 & $13 \%$ & 97 & $23 \%$ & 138 & $4 \%$ & 27 & $17 \%$ & 64 & $2 \%$ & 50 & $2 \%$ & 129 & $23 \%$ & 4,688 \\
\hline K54 × Basmati 217 & $52 \%$ & 82 & $33 \%$ & 135 & $30 \%$ & 29 & $78 \%$ & 61 & $40 \%$ & 49 & $7 \%$ & 128 & $21 \%$ & 4,680 \\
\hline K9 9 PAN 84 & $7 \%$ & 87 & $6 \%$ & 121 & $-4 \%$ & 23 & $-22 \%$ & 16 & $-33 \%$ & 15 & $19 \%$ & 123 & $-10 \%$ & 5,680 \\
\hline $\mathrm{K} 8 \times$ PAN 84 & $5 \%$ & 82 & $4 \%$ & 123 & $-9 \%$ & 24 & $-12 \%$ & 17 & $-14 \%$ & 16 & $8 \%$ & 124 & $0 \%$ & 5,680 \\
\hline K54 × PAN 84 & $-5 \%$ & 87 & $2 \%$ & 122 & $-4 \%$ & 22 & $-24 \%$ & 18 & $-35 \%$ & 14 & $-7 \%$ & 126 & $-27 \%$ & 4,967 \\
\hline K9 $\times$ Dourado precoce & $0 \%$ & 85 & $12 \%$ & 130 & $9 \%$ & 27 & $-24 \%$ & 28 & $-30 \%$ & 19 & $2 \%$ & 130 & $-16 \%$ & 4,686 \\
\hline K8 $\times$ Dourado precoce & $2 \%$ & 82 & $7 \%$ & 129 & $-9 \%$ & 26 & $-2 \%$ & 31 & $-12 \%$ & 20 & $-13 \%$ & 129 & $-28 \%$ & 4,683 \\
\hline K54 $\times$ Dourado precoce & $17 \%$ & 82 & $-4 \%$ & 131 & $-22 \%$ & 28 & $-24 \%$ & 29 & $-49 \%$ & 18 & $2 \%$ & 127 & $20 \%$ & 5,680 \\
\hline K9 $\times$ Komboka & $7 \%$ & 102 & $19 \%$ & 141 & $0 \%$ & 20 & $90 \%$ & 51 & $70 \%$ & 34 & $23 \%$ & 116 & $24 \%$ & 3,682 \\
\hline $\mathrm{K} 8 \times$ Komboka & $55 \%$ & 99 & $32 \%$ & 139 & $-9 \%$ & 21 & $29 \%$ & 50 & $2 \%$ & 36 & $4 \%$ & 115 & $-26 \%$ & 3,910 \\
\hline K54 $\times$ Komboka & $37 \%$ & 82 & $24 \%$ & 142 & $-23 \%$ & 20 & $27 \%$ & 52 & $-23 \%$ & 37 & $8 \%$ & 114 & $-12 \%$ & 3,680 \\
\hline K9 × IR-27-93-80-1 & $16 \%$ & 118 & $21 \%$ & 157 & $-9 \%$ & 21 & $15 \%$ & 61 & $2 \%$ & 43 & $9 \%$ & 107 & $-23 \%$ & 3,167 \\
\hline K8 $\times$ IR-27-93-80-1 & $5 \%$ & 112 & $13 \%$ & 156 & $-4 \%$ & 23 & $37 \%$ & 60 & $9 \%$ & 44 & $3 \%$ & 110 & $-18 \%$ & 3,085 \\
\hline K54 × IR-27-93-80-1 & $-4 \%$ & 82 & $3 \%$ & 158 & $-4 \%$ & 22 & $17 \%$ & 62 & $-5 \%$ & 43 & $4 \%$ & 109 & $-32 \%$ & 3,680 \\
\hline
\end{tabular}

\section{Discussion}

\subsection{Estimate of Genetic Components}

The analysis of variance for combining ability showed that the rice genotypes differed significantly for all the agronomic and yield traits studied. Further analysis of GCA/SCA variances showed that the nature of gene action was additive due to high magnitude of fixable genetic component for days to $50 \%$ flowering, days to maturity, number of tillers plant ${ }^{-1}$, number of spikelet's panicle ${ }^{-1}$, number of effective tillers plant ${ }^{-1}$, number of fertile grains panicle $e^{-1}$ and grain yield. Non-additive gene action was dominant in plant height, panicle length, flag leaf length and 1000 grain weight. These results are contrary to the findings of Sathya and Jebaraj (2015) who reported dominance of non-additive gene action for all the traits studied under aerobic condition. 
Major role of non-additive gene effects in the inheritance of agronomic and yield related traits were reported (Muhammad et al., 2010; Hasan et al., 2013, 2015). Previous studies have reported predominant role of additive gene action in the control of yield traits (Faiz et al., 2006; Chakraborty et al., 2009; Satheesh et al., 2010; Gnanamalar \& Vivekanandan, 2013).

\subsection{Evaluation of Parents Based on GCA Effects}

The combining ability analysis revealed significant GCA and SCA variance for most of the agronomic characters studied suggesting the importance of both additive and non-additive gene actions in the expression of the yield traits. Previous studies reported the significance of both additive and non-additive gene action in the expression of agronomic and yield traits (Thakare et al., 2009; Mirarab et al., 2011; Padmavathi et al., 2012; Dar et al., 2014 and Malemba et al., 2017). The rice parents such as Basmati 370, Basmati 217, K2-54 and Komboka had high and positive GCA effects for grain yield suggesting that these parental genotypes were good general combiners for grain yield. Previous studies also reported good general combiners for yield and yield traits in rice genotypes (Padmavathi et al., 2012; Raju et al., 2014; Sathya \& Jebaraj, 2015).

The parental lines such as Basmati 370, Basmati 217, PAN 84 and Komboka exhibited high and positive GCA effects for panicle length, flag leaf length, number of tillers plant ${ }^{-1}$, number of spikelet's panicle ${ }^{-1}$, number of panicles plant ${ }^{-1}$ and number of filled grains panicle ${ }^{-1}$ suggesting their desirability for improvement of positive agronomic and yield traits because they contributed to high grain yield, in contrast parents with low and negative GCA estimates (NERICA 1, PAN 84, $\mathrm{K}_{2}-9$, Dourado precoce and $\mathrm{K}_{2}-54$ ) are preferred for improvement of negative traits of grain yield such as plant height, $50 \%$ days to flowering and days to maturity. Previous studies reported the significance of using parents with high and positive GCA effects for the improvement of positive agronomic traits (Mirarab et al., 2011; Raju et al., 2014; Malemba et al., 2017). This study revealed high GCA variances for most of the yield traits studied. Mirarab et al. (2011) conducted a study on heterosis, combining ability and genetic parameters of yield and yield components in rice. They reported that GCA was only significant for total number of kernels per panicle, number of filled grains and grain yield per plant implying that additive gene action was predominant in the control of these traits. Previous research also reported the predominance of additive gene action in the inheritance of days to maturity, number of productive tillers plant-1, number of panicles per plant and 1000 grain weight (Dar et al., 2014). Therefore, it is suggested that a breeding method that would take care of the fixable gene effects and as well as maintain heterozygosity for exploiting the dominance effects may be more efficient for the improvement of grain yield (Chakraborty et al., 2009). Hence, a simple selection procedure followed by pedigree breeding is sufficient to improve the traits influenced by additive gene action (Chakraborty et al., 2009).

\subsection{Evaluation of $F_{1}$ Hybrids Based on SCA Effects}

The hybrids $\mathrm{K}_{2}-9 \times$ Komboka, $\mathrm{K}_{2}-9 \times$ Basmati $370, \mathrm{~K}_{2}-54 \times$ Dourado precoce and $\mathrm{K}_{2}-54 \times$ Basmati 217 were good specific combiners for grain yield because they expressed high SCA effects for grain yield. Similar studies also reported superior SCA effects for drought tolerant and yield traits in rice varieties (Muhammad et al., 2010; Sathya \& Jebaraj, 2015; Malemba et al., 2017). All the high yielding hybrids $\left(\mathrm{K}_{2}-9 \times \mathrm{Komboka}_{2} \mathrm{~K}_{2}-9 \times\right.$ Basmati $370, \mathrm{~K}_{2}-54 \times$ Dourado precoce and $\mathrm{K}_{2}-54 \times$ Basmati 217) originated from high $\times$ high GCA combinations attributable to additive $\times$ additive type of gene actions thus the yield potential of these hybrids can be fixed in subsequent generations (Sandhyakishore et al., 2011). However, negative SCA effects for grain yield were observed in the crosses $\mathrm{K}_{2}-9 \times$ NERICA $1, \mathrm{~K}_{2}-8 \times$ Dourado precoce and $\mathrm{K}_{2}-8 \times$ Komboka suggesting a low yield potential. The hybrid $\mathrm{K}_{2}-9 \times$ NERICA 1 originated from high $\times$ low GCA combination while the rest of the crosses with low and negative SCA effects originated from low $\times$ high GCA combinations. The high yield potential observed in the hybrids with high $\times$ low GCA combinations could be attributed to interaction between positive alleles in the good combiner and negative alleles in the poor combiner (Hasan et al., 2015). This study revealed that hybrids $\left(\mathrm{K}_{2}-54 \times\right.$ Basmati $217, \mathrm{~K}_{2}-54 \times$ Komboka, and $\mathrm{K}_{2}-54 \times$ Basmati 370$)$ that originated from high general combiner parents with high and positive SCA estimates are expected to produce desirable transgressive segregants which can be recognized by carrying out pedigree breeding method (Muhammad et al., 2010). High SCA effects of hybrids $\left(\mathrm{K}_{2}-9 \times\right.$ NERICA 1$)$ that originated from high $\times$ low GCA combining parents would be unfixable in subsequent generations and hence cannot be exploited by pedigree selection procedure (Sathya \& Jebaraj, 2015). But, in later generations these hybrids would produce desirable transgressive segregants upon modification of the conventional breeding methodology to accommodate both additive and non-additive genetic effects (Chakraborty et al., 2009). The present investigation revealed that parents with high GCA estimates were not always the best general combiners. Furthermore, the results also indicated that parents with high GCA effects were the best general combiners for only a specific trait but none of 
the parents or the specific crosses was best for all the characters studied. Similar findings were reported (Chakraborty et al., 2009; Malemba et al., 2017).

\subsection{Heterosis for Agronomic and Yield Components}

\subsubsection{Days to Maturity}

The hybrids $\mathrm{K}_{2}-54 \times$ Basmati $217, \mathrm{~K}_{2}-8 \times$ Komboka and $\mathrm{K}_{2}-54 \times$ SARO 5 expressed high parent heterosis for days to maturity while $\mathrm{K}_{2}-54 \times$ Basmati $370, \mathrm{~K}_{2}-54 \times$ Dourado precoce were early maturing over the better parent (NERICA 1). Previous studies reported negative heterosis for earliness in rice hybrids (Patil et al., 2011; Tiwari et al., 2011; Veeresha et al., 2013; Borah et al., 2017).

Positive heterosis for days to maturity was also reported (Nadali, 2010; Sunil Kumar et al., 2012). Development of early maturing and high yielding rice varieties are desired in rice breeding program (Rahimi et al., 2010). Negative heterosis is desirable for duration to $50 \%$ flowering and days to maturity (Borah et al., 2017). The hybrids with negative heterosis over better parent were early maturing. Therefore, the early maturing hybrids suggested the possibility of developing early maturing lines. Similar findings were also reported (Rahimi et al., 2010).

\subsubsection{Number of Tillers per Plant}

The crosses $\mathrm{K}_{2}-9 \times$ Komboka, $\mathrm{K}_{2}-54 \times$ Basmati 217 and $\mathrm{K}_{2}-54 \times$ Basmati 370 had higher better parent heterosis while $\mathrm{K}_{2}-54 \times$ Dourado precoce and $\mathrm{K}_{2}-9 \times$ PAN 84 showed negative heterosis for number of tillers. Patil et al., (2011), Tiwari et al., (2011) and Sunil Kumar et al., (2012) reported significant and desirable heterosis for effective tillers over better parent. Panicle bearing tillers have a strong correlation with grain yield thus leading to high crop productivity. Therefore, positive heterosis is desirable for effective tiller plant ${ }^{-1}$ (Borah et al., 2017).

In this study, high parent heterosis for effective tillers was observed in $16 F_{1}$ hybrids suggesting that these $F_{1}$ hybrids had high number of effective tillers and thus possessed high yield potential. Previous studies reported high parent heterosis for number of tillers in $\mathrm{F}_{1}$ rice hybrids (Mirarab et al., 2011; Dwivedi \& Pandey, 2012; Borah et al., 2017).

\subsubsection{Panicle Length}

The crosses $\mathrm{K}_{2}-8 \times$ Basmati 370, $\mathrm{K}_{2}-54 \times$ Basmati 370, $\mathrm{K}_{2}-9 \times$ Basmati 217, $\mathrm{K}_{2}-9 \times$ Basmati 217 and $\mathrm{K}_{2}-8 \times$ Basmati 370 showed high parent heterosis for panicle length while $\mathrm{K}_{2}-54 \times$ Komboka and $\mathrm{K}_{2}-54 \times$ Dourado precoce exhibited negative heterosis for panicle length. Patil et al., (2011) reported significant heterobeltiosis of up to $24.06 \%$ for panicle length. Previous studies reported both positive and negative heterosis for panicle length (Singh et al., 1992; Tiwari et al., 2011; Bhati et al., 2015). Large panicles are normally associated with high number of grains panicle-1 resulting in high grains yields (Bhati et al., 2015). Therefore, hybrids with positive heterosis for panicle length are desirable.

\subsubsection{Filled Grains per Panicle}

The hybrids $\mathrm{K}_{2}-54 \times$ Basmati $370, \mathrm{~K}_{2}-9 \times$ Komboka and $\mathrm{K}_{2}-9 \times$ PAN 84 had high parent heterosis for filled grains per panicle while $\mathrm{K}_{2}-8 \times$ Basmati 370 and $\mathrm{K}_{2}-8 \times 08 \mathrm{FAN} 10$ had negative heterosis. These results are contrary to the findings of Mirarab et al. (2011) who reported non-significant heterobeltiosis for filled grains per panicle. Positive heterobeltiosis in the hybrid IR58025A $\times$ IET18648 (49.73\%) and standard heterosis of $141.1 \%$ over the check variety TTB404 for filled grains panicle ${ }^{-1}$ was reported (Tiwari et al., 2011; Borah et al., 2017). The number of fertile grains panicle ${ }^{-1}$ is directly associated to grain yield and therefore a positive heterosis would be desirable in order to increase the productivity of rice varieties (Tiwari et al., 2011). In this study, $15 \mathrm{~F}_{1}$ hybrids were superior in terms of filled grains per panicle over the better parent implying that these hybrids were high yielding because fertile grains per panicle has a direct association with grain yield.

\subsubsection{Grain Yield}

The crosses $\mathrm{K}_{2}-9 \times$ Basmati 370, $\mathrm{K}_{2}-9 \times$ Komboka, $\mathrm{K}_{2}-8 \times$ Basmati 217, $\mathrm{K}_{2}-54 \times$ Basmati 217 and $\mathrm{K}_{2}-54 \times$ Dourado precoce were high grain yielders while $\mathrm{K}_{2}-9 \times$ NERICA $1, \mathrm{~K}_{2}-9 \times$ SARO 5 and $\mathrm{K}_{2}-9 \times$ PAN 84 had negative heterosis for grain yield. Borah et al., (2017) reported negative heterosis in some $F_{1}$ rice hybrids. Previous studies reported high parent heterosis for grain yield in rice hybrids (Faiz et al., 2006; Rahimi et al., 2010; Mirarab et al., 2011; Dwivedi \& Pandey, 2012; Bhati et al., 2015). Hybrid vigour for grain yield was due to interaction of many yield components such as days to maturity, panicle length, number of tillers plant ${ }^{-1}$, number of panicles plant ${ }^{-1}$ and filled grains panicle ${ }^{-1}$ (Veeresha et al., 2015). Grafius (1959) reported that grain yield is a complex trait and it is a multiplicative end product of several yield components. In this study, crosses with hybrid vigour for grain yield exhibited heterotic effect along with hybrid vigour for panicle length, number 
tillers plant ${ }^{-1}$, number of panicles plant ${ }^{-1}$ and fertile grains panicle ${ }^{-1}$. Swaminathan et al. (1972) reported that heterobeltiosis of more than $20 \%$ over better parent could offset the cost of hybrid seed production. Therefore, the hybrids $\mathrm{K}_{2}-9 \times$ Basmati $370, \mathrm{~K}_{2}-8 \times$ Basmati 217, $\mathrm{K}_{2}-54 \times$ Basmati 217 and $\mathrm{K}_{2}-9 \times$ Komboka were high grain yielders compared to the check variety thus they can be utilized for hybrid rice production.

\section{Conclusions and Recommendations}

The analysis of combining ability and heterosis for agronomic and yield related traits between indica and japonica rice crosses showed that the parental lines Basmati 370, Basmati 217, $\mathrm{K}_{2}-54$ and Komboka were good general combiners for grain yield and offered good scope for developing rice hybrids with high yield potential. In contrast, their crosses $\mathrm{K}_{2}-9 \times$ Komboka, $\mathrm{K}_{2}-9 \times$ Basmati $370, \mathrm{~K}_{2}-54 \times$ Dourado precoce and $\mathrm{K}_{2}-54 \times$ Basmati 217 were good specific combiners and expressed better heterotic effects for grain yield. Days to $50 \%$ flowering, days to maturity, number of tillers plant ${ }^{-1}$, number of spikelet's panicle ${ }^{-1}$, number of effective tillers plant ${ }^{-1}$, number of fertile grains panicle ${ }^{-1}$ and grain yield were governed by additive gene action suggesting that hybridization followed by selection in later generations may be recommended for improvement of these traits. Non-additive gene action was dominant in plant height, panicle length, flag leaf length and 1000 grain weight implying that the genetic potential of these traits would be unfixable in subsequent generations but modification of the pedigree breeding procedure to accommodate the non-additive genetic effects may be recommended.

\section{Acknowledgements}

This research was supported by Alliance for a Green Revolution in Africa (AGRA) Grant No. 2015 PASS 011. The opinions expressed herein are those of the author(s) and do not necessarily reflect the views of AGRA. The author(s) are thankful to the Industrial Crops Research Centre of the Kenya Agricultural and Livestock Research Organization for providing germplasm and the infrastructural support.

\section{References}

ARC (Africa Rice Center). (2008). NERICA: The New Rice for Africa-A Compendium. In E. A. Somado, R. G. Guei, \& S. O. Seya (Eds.), Cotonou Benin: Africa Rice Center (WARDA) (p. 210). Rome, Italy: FAO; Tokyo, Japan: Sasakawa Africa Association.

Alam, M. F., Khan, M. R., Nuruzzaman, M., Parvez, S., Swaraz, M. A., Alam, I., \& Ahsan, N. (2004). Genetic basis of heterosis and inbreeding depression in rice (Oryza sativa L.). Journal of Zhejiang University of Science, 5(1), 406-411. http://doi.org/10.1631/jzus.2004.0406

Baker, R. J. (1978). Issues in diallel analysis. Journal of Crop Science, 18(4), 533-536. http://doi.org/10.2135/ cropsci1978.0011183X001800040001x

Bhati, P. K., Singh, S. K., Singh, R., Sharma, A., \& Dhurai, S. Y. (2015). Estimation of heterosis for yield and yield related traits in rice (Oryza sativa L.). SABRAO Journal of Breeding and Genetics, 47(4), 467-474.

Borah, P., Sarma, D., \& Hazarika, G. N. (2017). Magnitude of heterosis for yield and its components in hybrid rice (Oryza sativa L.). International Journal of Agricultural Science and Research, 7(2), 209-216. https://doi.org/10.24247/ijasrapr201729

Chakraborty, R., Chakraborty, S., Dutta, B. K., \& Paul, S. B. (2009). Combining ability analysis for yield and yield components in bold grained rice (Oryza sativa L.) of Assam. PALIMIRA, 58(1), 9-13.

Chaudhary, R. C. (1984). Introduction to Plant Breeding. New Delhi, Oxford and IBH.

Coffman, R., \& Herrera, R. (1980). In R. A. Forsberg, A. R. Hallauer, \& A. W. Hovin (Eds.), Hybridization of Crop Plants. Wisconsin, USA: The American Society of Agronomy, Inc., and the Crop Science Society of America, Inc.

Comstock, R. E., \& Robinson, H. F. (1948). The components of genetic variance in populations of biparental progenies and their uses in estimating the average degree of dominance. Biometrics, 4(4), 254-266. https://doi.org/10.2307/3001412

Comstock, R. E., \& Robinson, H. F. (1952). In J. W. Gowen (Ed.), Estimation of average dominance of genes: Heterosis (pp. 494-515). Iowa State College Press-Ames.

Dar, S., Rather, A. G., Ahanger, M. A., Sofi, N. R., \& Talib, S. (2014). Gene action and combining ability studies for yield and component traits in rice (Oryza sativa L.): A review. Journal of Plant and Pest Science, 1(3), 110-127.

Dogara, A. M., \& Jumare, A. I. (2014). Origin, distribution and heading date in cultivated rice. International Journal of Plant Biology and Research, 2(1), 1-6. 
Dwivedi, D. K., \& Pandey, M. P. (2012). Gene action and heterosis for yield and associated traits in indica and tropical japonica crosses of rice involving wide compatibility genes. International Journal of Plant Breeding and Genetics, 6(3), 140-150. http://doi.org/10.3923/ijpbj.2012.140.150

Faiz, F. A., Sabar, M., Awan, T. H., Ijaz, M., \& Manzoor, Z. (2006). Heterosis and combining ability analysis in Basmati rice hybrids. Journal of Animal and Plant Science, 16(2), 56-59.

Fasahat, P., Abazar, R., Javad, M. R., \& John, D. (2016). Principles and Utilization of Combining Ability in Plant Breeding. Biometrics and Biostatistics International Journal, 4(1), 1-24. https://doi.org/10.15406/bbij.2016. 04.00085

FAO (Food and Agricultural Organization). (2017). Rice market monitor (pp. 1-42). Bangkok, Thailand. Retrieved from http://www.fao.org/economic/RMM

Gnanamalar, R. P., \& Vivekanandan, P. (2013). Combining ability analysis of grain quality traits in rice (Oryza sativa L.). Asian Journal of Plant Science and Research, 3(2), 145-149.

Gomez, K. A. (1972). Techniques for field experiments with rice. Manila, Philippines: International Rice Research Institute. Retrieved from http://www.nal.usda.gov

Grafius, J. E. (1959). Heterosis in barley. Journal of Agronomy, 51(1), 551-554. https://doi.org/10.2134/ agronj1959.00021962005100090013x

Gregorio, A., Angela, P., Juan, B., \& Jose, C. (2015). Analysis of genetic designs in R. Mexico: International Maize and Wheat Improvement Centre. Retrieved from http://www.gnu.org/licenses/gpl-3.0.html

Griffing, B. (1956). Concept of general and specific combining ability in relation to diallel crossing system. Australian Journal of Biological Sciences, 9(4), 463-493. https://doi.org/10.1071/BI9560463

Hasan, M. J., Kulsum, M. U., Hossain, E., Hossain, M. M., Rahman, M. M., \& Rahmat, F. M. N. (2015). Combining ability analysis for identifying elite parents for heterotic rice hybrids. Academia Journal of Agricultural Research, 3(5), 70-75.

Hasan, M. J., Kulsum, U. K., Lipi, L. F., \& Shamsuddin, A. K. M. (2013). Combining ability studies for developing new rice hybrids in Bangladesh. Bangladesh Journal of Botany, 42(2), 215-222.

IRRI (International Rice Research Institute). (2013). Standard Evaluation System (SES) for Rice (5th ed.). Los Banos, Philippines.

Kempthrone, D., \& Curnow, R. N. (1961). The partial diallel cross. Biometrics, 17(2), 229-240. https://doi.org/ $10.2307 / 2527989$

Kempthrone, O. (1957). An Introduction of Genetics Statistics. New York, USA: Wiley and Sons. Retrieved from http://psycnet.apa.org/psycinfo/1958-01083-000

KARI (Kenya Agricultural Research Institute). (2000). Soil fertility management handbook for extension staff and farmers in Kenya. KARI Technical Note Series (p. 45).

Kimani, J. M. (2010). Genetic studies of quantitative and quality traits in rice under low and high soil nitrogen and phosphorous conditions, and a survey of farmer preferences for cultivars (Unpublished $\mathrm{PhD}$ thesis, University of KwaZulu-Natal, Republic of South Africa).

Malemba, G. M., Nzuve, F. M., Kimani, J. M., Olubayo, M. F., \& Muthomi, J. W. (2017). Combining ability for drought tolerance in upland rice varieties at reproductive stage. Journal of Agricultural Science, 9(3), 138-150. https://doi.org/10.5539/jas.v9n3p138

MoA (Ministry of Agriculture). (2014). National rice development strategy 2008-2018. Agriculture Information Center, Nairobi, Kenya.

Mirarab, M., Ahmadikhah, A., \& Pahlavani, M. H. (2011). Study on combining ability, heterosis and genetic parameters of yield traits in rice. African Journal of Biotechnology, 10(59), 12512-12519. https://doi.org/ $10.5897 /$ AJB11.501

Muhammad, Y. S., Javed, I. M., \& Muhammad, A. H. (2010). Combining ability analysis of some morpho-physiological traits in Basmati rice. Pakistan Journal of Botany, 42(5), 3113-3123.

Nadali, B. (2010). Heterosis and combining ability analysis for yield and related-yield traits in hybrid rice. International Journal of Biology, 2(2), 222-231. 
Njiruh, P. N., Kanya, J. I., Kimani, J. M., \& Wajogu, R. K. (2013). Production of hybrid Basmati rice in Kenya: Progress and Challenges. International Journal of Innovations in Bio-Sciences, 3(4), 115-124.

Padmavathi, P. V., Satyanarayana, P. V., Lal Ahamed, M., Rani, A., \& Rao, V. S. (2012). Combining ability studies for yield and yield components trait in hybrid rice (Oryza sativa L.). Electronic Journal of Plant Breeding, 3(3), 836-842.

Patil, P. P., Vashi, R. D., Shinde, D. A., \& Lodam, V. A. (2011). Nature and magnitude of heterosis for grain yield and yield attributing traits in rice (Oryza sativa L.) Plant Archives, 11(1), 423-427.

Rahimi, M., Rabiei, B., Samizadeh, H., \& Ghasemi, A. K. (2010). Combining ability and heterosis in rice (Oryza sativa L.) cultivars. Journal of Agricultural Science and Technology, 12(1), 223-231.

Raju, C. D., Kumar, S. S., Raju, C. S., and Srijan, A. (2014). Combining ability studies in the selected parents and hybrids in rice. International Journal of Pure and Applied Bioscience, 2(4), 271-279.

Sandhyakishore, N., Ramesha, D., Reddy, M. S. T., \& Hari Prasad, A. S. (2011). Combining ability analysis for identifying elite parents for heterotic rice hybrids. Oryza, 48, 101-107.

Satheesh, P., Saravanan, K., \& Sabesan, T. (2010). Combining ability for yield and yield contributing characters in rice (Oryza sativa L.). Electronic Journal of Plant Breeding, 1(5), 1290-1293.

Sathya, R., \& Jebaraj, S. (2015). Combining ability analysis of three line hybrids in rice (Oryza sativa L.) under aerobic condition. International Journal of Plant Sciences, 10(2), 122-129. https://doi.org/10.15740/ HAS/IJPS/10.2/122-129

Singh, A. K., Maurya, D. M., \& Giri, S. P. (1992). Estimation of heterosis in rice. Oryza, 29(1), 259-261.

Sunil Kumar, Satish, K., Narendra, P., \& Dwivedi, D. K. (2012). Estimation of heterosis for grain yield and yield attributing traits in rice (Oryza sativa L.) Plant Archives, 12(1), 159-164.

Swaminathan, M. S., Siddiq, E. A., \& Sharma, S. D. (1972). Outlook for hybrid rice in India: In rice breeding (pp. 609-613). Manila Philippines: International Rice Research Institute.

Syed, E., \& Khaliq, I. (2008). Quantitative inheritance of some physiological traits for spring wheat under two different population densities. Pakistan Journal of Botany, 40(2), 581-587.

Thakare, I. S., Mehta, A. M., Patel, J. S., \& Takle, S. R. (2009). Combining ability analysis for yield and grain quality traits in rice hybrids. Journal of Rice Research, 3(1), 1-5.

Tiwari, D. K., Pandey, P., Giri, S. P., \& Dwivedi, L. J. (2011). Heterosis studies for yield and its components in rice hybrids using CMS system. Asian Journal of Plant Science, 10(1), 29-42. https://doi.org/10.3923/ ajps.2011.29.42

Vanaja, T., \& Babu, L. C. (2004). Heterosis for yield and yield components in rice (Oryza sativa L.). Journal of Tropical Agriculture, 42(1), 43-44.

Veeresha, B. A., Hanamaratti, N. G., Salimath, P. M., \& Chetti, M. B. (2013a). Heterosis for yield and yield traits in hybrid rice (Oryza sativa L.) under aerobic condition. Bioinfolet., 10(2A), 521-529.

Veeresha, B. A., Hanamaratti, N. G., \& Salimath, P. M. (2015). Heterosis and combining ability studies for yield and productivity traits in rice: A Review. International Journal of Current Agricultural Research, 4(5), 120-126.

Virmani, S. S., Viraktamath, B. C., Casal, C. L., Toledo, R. S., Lopez, M. T., \& Manalo, J. O. (1997). Hybrid rice breeding manual (p. 4). Los Banos, Philippines.

\section{Copyrights}

Copyright for this article is retained by the author(s), with first publication rights granted to the journal.

This is an open-access article distributed under the terms and conditions of the Creative Commons Attribution license (http://creativecommons.org/licenses/by/4.0/). 